\title{
Increased in Vitro Release of Interferon-Gamma from Ampicillin- Stimulated Peripheral Blood Mononuclear Cells in Stevens-Johnson
} Syndrome

\author{
Tomoaki Yoshimura, * Emi Hamaguchi, Eiseki Usami, Keiji Nakashima, Mayumi Kawaguchi, \\ Norio Suzuki, Yoshimi Oкамото, Toshiya NaKao, and Futoshi Yamazaki \\ Department of Pharmacy, Ogaki Municipal Hospital; 4-86 Minaminokawa-cho, Ogaki, Gifu 503-8502, Japan. \\ Received November 14, 2003; accepted February 16, 2004
}

Stevens-Johnson syndrome (SJS) was diagnosed in a 39-year-old woman, treated with ampicillin (4000$8000 \mathrm{mg}$ daily), phenytoin ( $250 \mathrm{mg}$ daily), and furosemide ( $20-40 \mathrm{mg}$ daily) for 25,21 , and $20 \mathrm{~d}$, respectively, before the appearance of the eruption. The lymphocyte stimulation test with the MTT [3-(4,5-dimethyl thiazol-2yl)-2,5-diphenyl tetrazolium bromide] assay on ampicillin, phenytoin, and furosemide showed a low value of stimulation index, which indicated negative reactivity. An in vitro IFN- $\gamma$ release test was conducted on the patient with SJS and on two healthy controls. IFN- $\gamma$ release increased by $52 \%$ following the in vitro challenge of the patient's peripheral blood mononuclear cells (PBMCs) with $15 \mu \mathrm{g} / \mathrm{ml}$ of ampicillin, but not with phenytoin or furosemide. Neither of the controls experienced increased IFN- $\gamma$ release. In the present case, increased in vitro IFN- $\gamma$ release was observed on ampicillin-stimulated PBMCs, which may indicate the role of ampicillin as the drug responsible for the induction of SJS, and may imply the role of IFN- $\gamma$ in the pathogenesis of SJS.

Key words Stevens-Johnson syndrome; interferon- $\gamma$; ampicillin; in vitro

Many drugs, including antibiotics and antiepileptic drugs, are associated with severe adverse cutaneous reactions such as Stevens-Johnson syndrome (SJS) and toxic epidermal necrolysis (TEN). SJS is characterized by atypical target lesions accompanied by widespread blisters involving the face, trunk and extremities, in association with ocular and mucous membrane manifestations. ${ }^{1,2)}$ The pathogenesis of SJS is still unclear; however, immunological mechanisms, particularly $\mathrm{T}$ cell-dependent reactions, such as cell-mediated cytotoxicity, have been proposed. ${ }^{1)}$ The phenotype of the responding Tcells and their cytokine pattern might correlate with the type of immune response. IFN- $\gamma$ acts as a regulator of antigen presentation and of proliferation and differentiation of lymphocyte populations in the subsequent antigen-specific phase of the immune response. ${ }^{3)}$ IFN- $\gamma$ promotes Th1 responses, including cell-mediated cytotoxicity, so it may be involved in the pathogenesis of SJS.

Adverse cutaneous reactions to drugs mediated by an immunological mechanism are a common problem in daily medical practice. The evaluation of a drug-induced event and the identification of the offending drug is often a difficult task. In vitro tests based on drug-related T-cell activity, such as the lymphocyte stimulation test (LST), macrophage or leukocyte migration inhibition test, a correlation of delayedtype hypersensitivity and cell-mediated immunity, have been used as a diagnostic tool in various types of adverse drug reactions. Some studies related to drug-specific T-cell clones derived from the peripheral blood mononuclear cells (PBMCs) of patients with cutaneous drug reactions, revealed the release of Th1-type cytokines, including IFN- $\gamma$. The diagnostic role of in vitro IFN- $\gamma$ release has been reported in cases of allopurinol-induced SJS, ${ }^{4)}$ carbamazepine-induced erythroderma, ${ }^{5}$ paracetamol and bromhexine-induced acute generalized exanthematous pustulosis, ${ }^{6}$ ) and betalactam-induced morbilliform exanthems. ${ }^{7)}$ An increased expression of IFN- $\gamma$ was detected in bacampicillin-treated PBMCs from allergic contact dermatitis, but not from rhinitis patients. ${ }^{8)} \mathrm{Sim}$ - ilarly, IFN- $\gamma$ release and mRNA induction have been reported in penicillin allergies manifested by urticaria, angioedema and exanthem, ${ }^{9)}$ and in betalactam antibiotic-induced hypersensitivity. ${ }^{10)}$ In vitro cytokine release may have a diagnostic role in cutaneous drug reactions.

In this study, we present a patient developing SJS during treatment with ampicillin, phenytoin, and furosemide, in whom increased in vitro IFN- $\gamma$ release from ampicillin-stimulated PBMCs was observed.

\section{MATERIALS AND METHODS}

Materials RPMI 1640 culture medium and phosphatebuffered saline (PBS) (-) were commercially obtained from Nissui Pharmaceutical Co., Ltd.; fetal bovine serum (FBS) was from Gibco Life Technologies Co.; lymphocyte separation medium was from Organon Teknika Co.; phytohemagglutinin (PHA) was from Difco Laboratories; 3-(4,5-dimethyl thiazol-2-yl)-2,5-diphenyl tetrazolium bromide (MTT) and dimethylsulfoxide (DMSO) were from Dojin Chemicals.

An enzyme-linked immunosorbent assay (ELISA) kit for human IFN- $\gamma$ was obtained from Medgenix Diagnostics. A Fujirebio MPR A4i microplate reader was used for the assays.

Methods. Preparation of Peripheral Blood Mononuclear Cells PBMCs were obtained from heparinized peripheral blood from donors using a lymphocyte separation medium. The cells were then washed with PBS $(-)$ and resuspended in RPMI 1640 medium supplemented with $10 \%$ heat-inactivated FBS. They were then cultured at $37{ }^{\circ} \mathrm{C}$ in a humidified atmosphere of $5 \% \mathrm{CO}_{2} / 95 \%$ air.

Lymphocyte Stimulation Test with MTT Assay PBMC $\left(5 \times 10^{5}\right.$ cells $\left./ \mathrm{ml}\right)$ were incubated with or without various concentrations of the tested drugs. After $6 \mathrm{~d}$ of incubation, proliferative activity was determined by the MTT assay. ${ }^{11)}$ MTT was dissolved in PBS (-) to a concentration 
of $5 \mathrm{mg} / \mathrm{ml}$, and $50 \mu \mathrm{l}$ was added to each well of a 48 -well plate, after which incubation was performed for $4 \mathrm{~h}$ at $37^{\circ} \mathrm{C}$ in a humidified atmosphere of $5 \% \mathrm{CO}_{2} / 95 \%$ air. Then the plate was centrifuged for $15 \mathrm{~min}$ at $2000 \mathrm{rpm}$, the supernatant was removed, and $750 \mu \mathrm{l}$ of DMSO was added to dissolve the MTT formazan produced. When the formazan was fully dissolved, the absorption was measured at a wavelength of $540 \mathrm{~nm}$ and a reference wavelength of $620 \mathrm{~nm}$ using a microplate reader.

The lymphocyte blastogenesis was expressed by the stimulation index (SI): $\mathrm{SI}(\%)=\mathrm{A}_{2}-\mathrm{A}_{0} / \mathrm{A}_{1}-\mathrm{A}_{0} \times 100 . \mathrm{A}_{2}$ is the absorption of cultures with drugs added; $A_{1}$ is the absorption of cultures without drugs; $A_{0}$ is absorption of the blank (culture medium only).

Production and Assay of IFN- $\boldsymbol{\gamma}$ PBMC $\left(1 \times 10^{6}\right.$ cells $/ \mathrm{ml}$ ) were cultured for $72 \mathrm{~h}$ at $37^{\circ} \mathrm{C}$ in a 24 -well plate with tested drugs and $0.5 \mu \mathrm{g} / \mathrm{ml}$ PHA in a humidified atmosphere of $5 \% \mathrm{CO}_{2} / 95 \%$ air. Culture supernatant was collected by centrifugation at $2000 \mathrm{rpm}$ for $10 \mathrm{~min}$, and was stored at $-30{ }^{\circ} \mathrm{C}$ until use. The level of IFN- $\gamma$ was measured using a human IFN- $\gamma$ ELISA kit according to the manufacturer's recommendations. An IFN- $\gamma$ release test was performed in triplicate.

Statistical Analysis Each treatment in an individual experiment was carried out in triplicate. Results are expressed as the mean \pm standard error of the mean (S.E.M.). Data were analyzed using Student's paired $t$-test. Differences were considered significant at $p<0.05$.

Case Report A 39-year-old woman suffered from bacterial encephalitis and hydrocephalus after the treatment of acute myelocytic leukemia, and was taking ampicillin (4000 - 8000 mg daily), phenytoin (250 mg daily), and furosemide ( $20-40 \mathrm{mg}$ daily) for 25,21 , and $20 \mathrm{~d}$, respectively, before the appearance of the eruption. The patient exhibited widespread diffuse erythema, located on the trunk and limbs, and was diagnosed as erythema exsudativum multiform two days after the initial eruption. All drugs, as potentially responsible agents for the adverse cutaneous reaction, were discontinued at this time. A maculopapular eruption was observed to spread from the face and neck to the trunk and upper extremities, with bullous detachment of the epidermis in some areas on the trunk. Severe hemorrhagic bullous lesions on the lips, associated with erosion of the oral mucosa, and synechiae of the eyelids were observed. A diagnosis of SJS was made two days after the diagnosis of erythema exsudativum multiform. Supportive therapy, indicating systemic corticosteroids, antibiotics and nutrition support, resulted in complete resolution of the skin and mucous membrane lesions within one month.

Skin tests or challenge test with suspected drugs were not performed. However, the LST and an in vitro drug-induced IFN- $\gamma$ release test were performed on the patient with three suspected drugs, ampicillin, phenytoin, and furosemide, at the remission phase, $46 \mathrm{~d}$ after the appearance of the reaction.

\section{RESULTS}

The LST with the MTT assay and an in vitro IFN- $\gamma$ release test were performed on ampicillin, phenytoin, and furosemide. The drug concentrations used were 5, 15, and
$45 \mu \mathrm{g} / \mathrm{ml}$ for ampicillin, 3, 9 and $27 \mu \mathrm{g} / \mathrm{ml}$ for phenytoin, and 1,3 and $9 \mu \mathrm{g} / \mathrm{ml}$ for furosemide, which were extrapolated from the known therapeutic doses.

The results of the LST with the MTT assay are shown in Table 1. All drugs tested showed low values of stimulation index, which indicated negative reactivity.

An in vitro IFN- $\gamma$ release test was performed on the patient with SJS and on two healthy controls. IFN- $\gamma$ release increased by $52 \%$ following the in vitro challenge of the patient's PBMCs with $15 \mu \mathrm{g} / \mathrm{ml}$ of ampicillin, but not significantly $(p=0.054)$. On the other hand, phenytoin and furosemide showed almost no effect (Fig. 1). To investigate the effects of ampicillin, which showed an increase in IFN- $\gamma$ release in the patient, on PBMCs, an IFN- $\gamma$ release test was

Table 1. The LST with the MTT Assay against Suspected Drugs on a Patient with SJS

\begin{tabular}{ccc}
\hline \hline Drug & Conc. $(\mu \mathrm{g} / \mathrm{ml})$ & SI $(\%)$ \\
\hline \multirow{2}{*}{ Ampicillin } & 5 & 111 \\
& 15 & 113 \\
Phenytoin & 45 & 100 \\
& 3 & 105 \\
Furosemide & 9 & 110 \\
& 27 & 112 \\
& 1 & 106 \\
& 3 & 99
\end{tabular}

$\mathrm{SI}=$ stimulation index

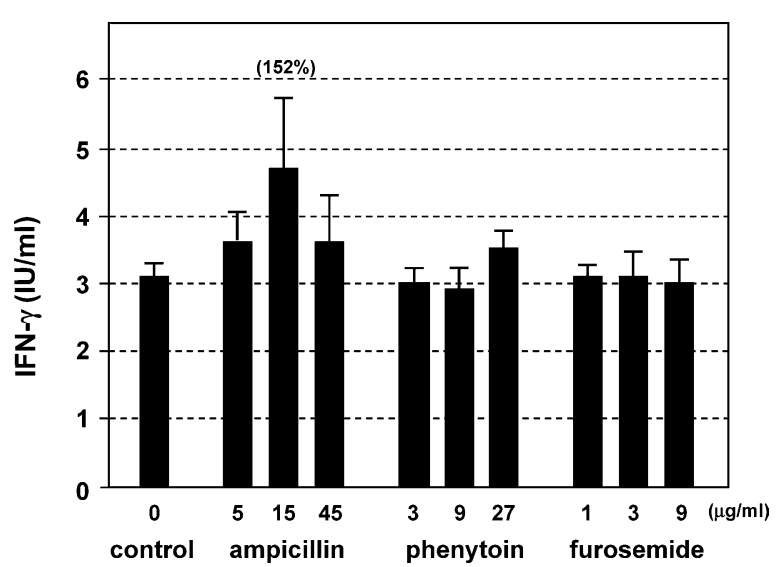

Fig. 1. In Vitro IFN- $\gamma$ Release from Suspected Drug-Stimulated PBMCs on a Patient with SJS

PBMCs $\left(1 \times 10^{6}\right.$ cells $\left./ \mathrm{ml}\right)$ were cultured for $72 \mathrm{~h}$ with tested drugs and $0.5 \mu \mathrm{g} / \mathrm{ml}$ PHA. The level of IFN- $\gamma$ in the culture supernatant was measured using a human IFN- $\gamma$ ELISA kit. Each column represents the mean \pm S.E.M. of triplicate experiments.

Table 2. Effects of Ampicillin on IFN- $\gamma$ Release from PBMCs on Healthy Controls

\begin{tabular}{lccc}
\hline \hline & Ampicillin $(\mu \mathrm{g} / \mathrm{ml})$ & IFN- $\gamma(\mathrm{IU} / \mathrm{ml})$ & $\%$ \\
\hline \multirow{2}{*}{ Control 1 } & 0 & $5.23 \pm 0.24$ & - \\
& 5 & $5.57 \pm 0.36$ & 107 \\
& 15 & $5.70 \pm 0.25$ & 109 \\
Control 2 & 45 & $5.13 \pm 0.18$ & 98 \\
& 0 & $4.07 \pm 0.10$ & - \\
& 5 & $4.52 \pm 0.23$ & 111 \\
& 15 & $4.40 \pm 0.27$ & 108 \\
& 45 & $4.10 \pm 0.18$ & 101 \\
\hline
\end{tabular}


performed on the healthy controls. Healthy controls were a 39-year-old man (control 1) and a 26-year-old woman (control 2) who did not suffer from immune-related disease. Ampicillin showed almost no effect on IFN- $\gamma$ release in the controls (Table 2).

\section{DISCUSSION}

Clinical evaluation of the agent causing adverse drug reactions may be complicated by factors such as simultaneous exposure to several drugs, drug interactions, and variability of the latent period between the administration of the suspected drug and the appearance of the reaction. Although the latency time is a key clinical feature to consider in an offending drug, it was similar $(25,21$, and $20 \mathrm{~d}$ for ampicillin, phenytoin, and furosemide, respectively), and all of drugs were discontinued at the same time in this case, which meant that it would be difficult to identify an offending drug only by clinical information. Both ampicillin and phenytoin are thought to be potential offending drugs because phenytoin and ampicillin have frequently been cited as causative agents of SJS. On the other hand, furosemide has not been reported as an offending drug in SJS. In case reports and studies, more than 100 drugs have been implicated as causes of SJS or TEN. ${ }^{1)}$ The frequency of allergic side effects has been correlated to the chemical structure of the drug. In this case, the causative drug might be ampicillin, since increased in vitro IFN- $\gamma$ release from ampicillin-stimulated PBMCs was observed. In recent these years, several reports of systemic reactions to betalactams due to type IV, cell-mediated, hypersensitivity mechanisms appeared in the literature. ${ }^{12,13)}$ Such reactions are often revealed by cutaneous symptoms, particularly after the administration of aminopenicillins.

IFN- $\gamma$ release increased by $52 \%$ following the in vitro challenge of the patient's PBMCs with $15 \mu \mathrm{g} / \mathrm{ml}$ of ampicillin. However, $45 \mu \mathrm{g} / \mathrm{ml}$ of ampicillin did not increase IFN$\gamma$ release, which indicated drug-induced IFN- $\gamma$ release did not operate in a dose-dependent manner. A proper range of antigen concentration may exist in an antigen-stimulated immune reaction. Ampicillin showed almost no effect on IFN- $\gamma$ release from PBMCs of the healthy controls. Increased IFN- $\gamma$ release from the patient's PBMCs indicated immune sensitization to the drug; however, further elucidation is needed to determine whether increasing by $52 \%$ in an IFN- $\gamma$ release test is adequate to diagnose a drug-induced adverse reaction.

We conducted the LST and an in vitro IFN- $\gamma$ release test at the remission phase, $46 \mathrm{~d}$ after the appearance of the reaction. Although performance of in vitro tests at the acute phase of the reaction is thought to be useful for the diagnosis, it is difficult to evaluate the function of lymphocytes, such as in cytokine production. One of the reasons arises from the administration of corticosteroids or immunosuppressive agents to treat the problem, which suppress the function of lymphocytes. Administration of such drugs would result in a weak reactivity of sensitized lymphocytes to the antigen in vitro tests. Other investigators also conducted in vitro tests $23 \mathrm{~d}$ or 2 months into the remission phase after resolution of the rash. ${ }^{4,6}$ One of the investigators described that performance of the in vitro test during the remission phase of SJS, rather than during the acute phase of the adverse reaction, may reduce the likelihood of a false positive IFN- $\gamma$ response for the offending drug. ${ }^{4)}$ The timing of the test is important for diagnosis. The reactivity of lymphocytes to the tested drug may differ at various stages of allergic symptoms.

In general, the LST is the only available in vitro test for detecting drug sensitization at the cellular level, irrespective of the effector mechanisms or clinical phenotype of the reaction, as it detects drug-specific proliferation of sensitized $\mathrm{T}$ cells. The LST with the MTT assay performed by us is a colorimetric assay that doesn't use radioactive substances. ${ }^{11)}$ Despite its known disadvantages, the LST gave positive results with a variety of drugs, including antibiotics and anticonvulsant agents. Drug-specific lymphocyte activation of the suspected drugs could not be detected in the patient presented here, as some authors argue that the LST may not be helpful in severe cutaneous drug reactions. ${ }^{14)}$ Conclusive diagnosis of drug allergy still remains a major problem in daily clinical practice. In vivo cutaneous tests like patch, prick and intracutaneous tests often do not yield positive reactions, even in well-documented histories of drug-allergic reactions. Final in vivo challenge tests, which are considered to be the most effective method, bear the risk of severe reactions, and are frequently not tolerated by the patients. In this case, in vivo challenge tests were not conducted for medicolegal reasons.

In the present case, increased in vitro IFN- $\gamma$ release was observed on ampicillin-stimulated PBMCs, which may indicate the role of ampicillin as the drug responsible for the induction of SJS, and may imply the role of IFN- $\gamma$ in the pathogenesis of SJS.

\section{REFERENCES}

1) Roujeau J. C., Stern R. S., N. Engl. J. Med., 331, 1272-1285 (1994).

2) Roujeau J. C., Kelly J. P., Naldi L., Rzany B., Stern R. S., Anderson T., Auquier A., Bastuji-Garin S., Correia O., Locati F., Mockenhaupt M., Paoletti C., Shapiro S., Shear N., Schopf E., Kaufman D. W., N. Engl. J. Med., 333, 1600-1607 (1995).

3) Billiau A., Heremans H., Vermeire K., Matthys P., Ann. N. Y. Acad. Sci., 856, 22-32 (1998).

4) Halevy S., Cohen A. D., Livni E., Int. J. Dermatol., 38, 835-840 (1999).

5) Koga T., Imayama S., Hori Y., Contact Dermatitis, 32, 181-182 (1995).

6) Halevy S., Cohen A. D. Livni E., Clin. Exp. Dermatol., 25, 652-654 (2000).

7) Hertl M., Geisel J., Boecker C., Merk H. F., Br. J. Dermatol., 128, 619-626 (1993).

8) Cederbrant K., Marcusson-Stahl M., Hultman P., Clin. Exp. Allergy, 30, 1450-1459 (2000).

9) Brander C., Mauri-Hellweg D., Bettens F., Rolli H., Goldman M., Pichler W. J., J. Immunol., 155, 2670-2678 (1995).

10) Gaspard I., Guinnepain M. T., Laurent J., Bachot N., Kerdine S., Bertoglio J., Pallardy M., Lebrec H., J. Clin. Immunol., 20, 107-116 (2000).

11) Yoshimura T., Kurita C., Yamazaki F., Nakano S., Nagai H., Biol. Pharm. Bull., 17, 921—926 (1994).

12) Vega J. M., Blanca M., Carmona M. J., Garcia J., Claros A., Juarez C., Moya M. C., Allergy, 46, 154-157 (1991).

13) Warrington R. J., Silviu-Dan F., Magro C., J. Allergy Clin. Immunol., 92, 626-628 (1993)

14) Shear N. H., Arch. Dermatol., 126, $94-97$ (1990). 